\title{
Circulating estrogens and postmenopausal ovarian cancer risk in the Women's Health Initiative Observational Study
}

\author{
Britton Trabert ${ }^{1}$, Louise A. Brinton ${ }^{1}$, Garnet L. Anderson ${ }^{2}$, Ruth M. Pfeiffer ${ }^{1}$, Roni T. Falk ${ }^{1}$, \\ Howard D. Strickler ${ }^{3}$, Sarunas Sliesoraitis ${ }^{4}$, Lewis H. Kuller ${ }^{5}$, Margery L. Gass ${ }^{6}$, Barbara J. \\ Fuhrman $^{7}, \mathrm{Xia} \mathrm{Xu}^{8}$, and Nicolas Wentzensen ${ }^{1}$
}

${ }^{1}$ Division of Cancer Epidemiology and Genetics, National Cancer Institute, Bethesda, Maryland 2Division of Public Health Sciences, Cancer Prevention Program, Fred Hutchinson Cancer Research Center, Seattle, Washington ${ }^{3}$ Department of Epidemiology and Population Health, Albert Einstein College of Medicine, Bronx, New York ${ }^{4}$ Department of Hematology and Medical Oncology, University of Texas at San Antonio, San Antonio, Texas ${ }^{5}$ Department of Epidemiology, University of Pittsburgh, Pittsburgh, Pennsylvania ${ }^{6}$ Women's Health Institute, Cleveland Clinic, Cleveland, Ohio, retired ${ }^{7}$ Department of Epidemiology, Fay W Boozman College of Public Health, University of Arkansas for Medical Sciences, Little Rock, Arkansas ${ }^{8}$ Cancer Research Technology Program, Leidos Biomedical Research, Inc., Frederick National Laboratory for Cancer Research, Frederick, Maryland

\section{Abstract}

Background-Hormonal and reproductive factors contribute to the development of ovarian cancer, but few studies have examined associations between circulating estrogens and estrogen metabolites and ovarian cancer risk. We evaluated whether serum estrogens and estrogen metabolite levels are associated with ovarian cancer risk among postmenopausal women in a nested case-control study in the Women's Health Initiative (WHI) Observational Study (OS).

Methods-We selected all 169 eligible epithelial ovarian cancer cases and 412 matched controls from women enrolled in WHI-OS who were not using menopausal hormones at baseline. Baseline levels of 15 estrogens and estrogen metabolites were measured via LC-MS/MS. Associations with ovarian cancer risk overall and stratified by histologic subtype (serous/non-serous) were analyzed using logistic regression. The mean time from serum collection to cancer diagnosis was 6.9 years.

Results-Overall we observed modest ovarian cancer risk associations among women with higher levels of estrone [Odds Ratio (95\% Confidence Interval) quintile (Q)5 vs. Q1: 1.54 (0.82$2.90)$, p-trend=0.05], as well as 2- and 4-methoxyestrone metabolites [2.03 (1.06-3.88), ptrend $=0.02 ; 1.86$ ( $0.98-3.56)$, p-trend $=0.01$, respectively]. Associations of estrogens and estrogen metabolites varied substantially by histologic subtype. Associations with serous tumors were

Corresponding author: Britton Trabert, 9609 Medical Center Drive, Bethesda, MD 20892-9774, Phone: 240-276-7331, Fax:

240-276-7838, ; Email: britton.trabert@ nih.gov.

Conflicts of interest: All authors declare they have no conflicts of interest

For a list of all the investigators who have contributed to WHI science, please visit: https://www.whi.org/researchers/Documents $\% 20 \% 20 \mathrm{Write} \% 20 \mathrm{a} \% 20 \mathrm{Paper} / \mathrm{WHI} \% 20$ Investigator\%20Long\%20List.pdf 
universally null, while estrone ( 2.65 (1.09-6.45), $\mathrm{p}$-trend $=0.01$, p-heterogeneity $=0.04)$, unconjugated estradiol (2.72 (1.04-7.14), p-trend $=0.03$, p-heterogeneity $=0.02)$ and many of the 2-, 4-, and 16-pathway metabolites were positively associated with non-serous tumors.

Conclusions-Our study provides novel molecular data showing an association of the parent estrogens and several estrogen metabolites with non-serous ovarian cancers.

Impact-These findings further support the heterogeneous etiology of ovarian cancer.

\section{Keywords}

Endogenous estrogens; estrogen metabolites; ovarian cancer risk; nested case-control study; heterogeneity

\section{INTRODUCTION}

Elevated estrogens may be involved in ovarian carcinogenesis; however, the exact nature of the estrogen-ovarian cancer link remains unclear (1). Studies indicated that ovarian cancer risk is higher in women with surrogates of high cumulative estrogen exposure including early age at menarche, late age at natural menopause, and long-duration exposures to menopausal hormone therapy (2-4). Following the marked reduction in menopausal hormone therapy use around 2002 there was an accelerated decrease in ovarian cancer incidence rates, further supporting a role for estrogens in postmenopausal ovarian cancer risk (5). Conversely, ovarian cancer risk is reduced in women with a history of oral contraceptive use, possibly by reducing the number of lifetime ovulations and by decreasing intra-ovarian estrogen levels (6).

In the premenopausal ovary, estrogen (namely estradiol) is produced via aromatization of androgens within the granulosa cells, and plays a significant role in follicular development (7). However in postmenopausal women, estrone is the predominant estrogen and is produced from peripheral conversion of adrenal androgens (7). Estrogen metabolites are formed from the conversion of estrone and estradiol via hydroxylation at the 2, 4, or 16 position of the carbon ring (Figure 1) (8). Estrogen effects, whether proliferative or antiproliferative, are modulated by metabolic conversions occurring systemically and in the target tissues (8). Most experimental research demonstrating differential effects of estrogen metabolites has been conducted in mammary tissue and breast cancer cell lines, with limited research on other hormonally-mediated tumors or their target tissues (8). The few experimental studies on ovarian cancer have demonstrated that estradiol can stimulate ovarian epithelial cell growth or induce aberrant proliferation with increasing DNA mutations (9-11), but have not evaluated similar effects of estrogen metabolites. The presence of sex steroid hormone receptors in malignant epithelial ovarian tumors supports a potential role for hormones in the origin and promotion of ovarian cancer (12).

Studies evaluating circulating estrogens and ovarian cancer risk are limited. Two prior studies that evaluated associations with pre-diagnostic circulating estrone and/or estradiol did not find associations with ovarian cancer risk $(13,14)$, and the one prior study that examined estrogen metabolites was not able to evaluate associations by subtype ( $\mathrm{n}=67$ ovarian cancer cases overall) and did not link pre-diagnostic estrogens to ovarian cancer 
(15). A study evaluating early pregnancy sex steroid hormones and subsequent cancer risk reported no associations with serous tumors, and an increased risk of endometrioid tumors with higher estradiol concentrations (16). Given the limited number of studies evaluating circulating estrogens and ovarian cancer risk, we conducted a nested case-control study within the Women's Health Initiative (WHI) Observational Study (OS), to evaluate the associations between 15 pre-diagnostic estrogens and estrogen metabolites and ovarian cancer risk among postmenopausal women not currently using menopausal hormones. With increasing evidence of etiologic heterogeneity of epithelial ovarian cancers-especially with respect to hormonal risk factors (e.g. body mass index (BMI), menopausal hormone therapy use) (17-19)—we also evaluated associations by tumor subtype.

\section{MATERIALS AND METHODS}

\section{Study population}

The Women's Health Initiative (WHI) is a large multicenter clinical investigation that enrolled women into either a clinical trial or an observational study to evaluate the predictors and causes of morbidity and mortality in postmenopause. The observational study (OS) is a prospective cohort that enrolled 93,676 postmenopausal women ages 50 to 79 years at 40 centers through the United States between 1993 and 1998 (20, 21). Women were excluded $(n=148)$ if they had medical conditions with a predicted survival of less than 3 years; if they had adherence/retention issues; or if they were participating in a clinical trial. The present nested case-control study included incident ovarian cancer cases that were diagnosed between study initiation and May 2012. Both cases and controls met the following criteria to be eligible: no history of cancer at baseline other than non-melanoma skin cancer ( $\mathrm{n}$ excluded=11,674); no current use of exogenous hormones $(38,419)$; no history of bilateral oophorectomy $(7,470)$; and at least $1.1 \mathrm{~mL}$ of available pre-diagnostic serum (720). Baseline serum samples were provided for all participants.

Ovarian cancer cases were WHI-OS participants with hospital-confirmed diagnoses of incident primary epithelial ovarian, fallopian tube, or peritoneal cancer. Primary ovarian cancers $(n=140)$ were locally and centrally adjudicated, while fallopian tube $(n=9)$ and peritoneal cancers $(n=20)$ were locally adjudicated. Among the cases, the mean time from sample collection to diagnosis was 6.9 years (standard deviation $=3.8$ years; range $=352$ days -14.8 years). Controls were eligible WHI-OS cohort members selected from strata defined by age at blood draw $(50-54,55-59,60-64,65-69,70-74,75-79)$, year at blood draw (1993-1996, 1997-1998), race/ethnicity (white, black, Hispanic, other/unknown), hysterectomy status at baseline or follow-up prior to event (yes/no), and time since last menopausal hormone use ( $\leq 1$ year, > 1 year). Controls were drawn from the set of eligible cohort members in each stratum containing ovarian cancer cases that were alive at the time of diagnosis of their matched case and were selected with a ratio of at least 2 controls per 1 case per stratum. We included never $(n=379)$ and former menopausal hormone users $(n=212$, of whom $n=18$ reported using hormones within 1 year of blood draw) in this analysis and excluded women with unconjugated estradiol concentrations greater than or equal to 184 $\mathrm{pmol} / \mathrm{L}(\sim 50 \mathrm{pg} / \mathrm{mL} ; \mathrm{n}=10)$ which is typically indicative of exogenous hormone use. The present study included 169 epithelial ovarian cancer cases and 412 matched controls. 
Among ovarian cancer cases, 102 were serous tumors and the remaining 67 were non-serous (13 endometrioid, 11 clear cell, 9 mucinous, and 34 other-epithelial subtypes). Approval for conducting the study was obtained from human subjects review at the Fred Hutchinson Cancer Research Center (WHI Clinical Coordinating Center), as well as at all 40 clinical centers. Written informed consent was obtained from study participants.

\section{Laboratory assays}

Stable isotope dilution LC-MS/MS was used to quantify 15 estrogens and estrogen metabolites including: estrone, estradiol, 2-pathway metabolites (2-hydroxyestrone, 2methoxyestrone, 2-hydroxyestradiol, 2-methoxyestradiol, and 2-hydroxyestrone-3-methyl ether); 4-pathway metabolites (4-hydroxyestrone, 4-methoxyestrone, and 4methoxyestradiol); and 16a -pathway metabolites (16a-hydroxyestrone, estriol, 16ketoestradiol, 16-epiestriol, and 17-epiestriol,). Details of the method have been published previously (22). For this study, six labeled internal standards were used: deuterated 2hydroxyestradiol, 2-methoxyestradiol and estriol (C/D/N Isotopes Inc, Pointe-Claire, QC, Canada); deuterated 16-epiestriol (Medical Isotopes Inc, Pelham, $\mathrm{NH}, \mathrm{USA}$ ); and ${ }^{13} \mathrm{C}$ labeled estrone and estradiol (Cambridge Isotope Laboratories, Andover, MA, USA).

In serum, this method detects 15 estrogens and estrogen metabolites which circulate, at least in part, as sulfated and/or glucuronidated conjugates to facilitate storage, transport, and excretion. Five of the estrogens (estrone, estradiol, estriol, 2-methoxyestrone and 2methoxyestradiol) were also measured in unconjugated forms in circulation. The serum sample was split into two aliquots, one to measure the combined concentration of each of the 15 metabolites (that is, the sum of conjugated plus unconjugated forms); the other, to measure the unconjugated forms. To measure the combined level of the conjugated plus unconjugated parent estrogen or estrogen metabolite level, an enzyme with sulfatase and glucuronidase activity was added to the samples to cleave any sulfate and glucoronide groups (22). To measure the unconjugated forms only, addition of the enzyme is not included in the sample preparation steps. For those metabolites with both combined and unconjugated measurements, the concentration of the conjugated form was calculated as the difference between the combined estrogen measurement and the unconjugated estrogen measurement, for estradiol that calculation was (conjugated E2 = combined E2 unconjugated E2). The limit of detection for each estrogen and estrogen metabolite measured using this LC-MS/MS assay was $10 \mathrm{fg}$ on column (approximately $0.33-0.37$ $\mathrm{pmol} / \mathrm{L})(22,23)$. There were no samples in the current study with undetectable levels for any of the hormones measured. Laboratory coefficients of variation (CV) of masked technical replicates across batches were $<6.0 \%$ for all hormones measured. Intraclass correlation coefficients (ICCs) ranged from 0.93-0.996 with median value of 0.98 .

\section{Statistical analysis}

Estrogens and estrogen metabolites were analyzed individually. Individual estrogens were categorized into quintiles based on the distribution in controls. First, we analyzed associations in all ovarian cancer cases (ovarian cancer overall), followed by analyses stratified by subtype (serous/non-serous). Conditional logistic regression models were used to estimate odds ratios (ORs) and 95\% confidence intervals (CIs) of ovarian cancer risk 
conditioning on matching factors: age at blood draw, calendar year of blood draw, race/ ethnicity, hysterectomy status, and time since last menopausal hormone use and further adjusted for a priori potential confounding factors: gravidity, BMI $(<25,25-29.9, \geq 30 \mathrm{~kg} /$ $\mathrm{m}^{2}$ ), cigarette smoking status (never, former, current), duration of oral contraception use (never, $<5,5-<10, \geq 10$ years) and menopausal hormone use (former/never). Tests for trend were based on the Wald statistic modeling the intra-category quintile median as a continuous parameter. Q-values reflecting the false discovery rates (FDR) were calculated to account for multiple comparisons in the main analysis and analyses by histologic subtype, all other analyses were considered exploratory and therefore not corrected for multiple comparisons. For analyses stratified by case characteristics we used baseline category polytomous logistic regression models, with the controls as the reference group and adjustment for matching factors and a priori selected potential confounding factors (listed above), with one exception: menopausal hormone therapy use and time since last menopausal hormone therapy use were combined (never, $\leq 1$ year, $>1$ year). We also evaluated associations stratified by time between blood collection and diagnosis ( $<5$ year, $\geq 5$ years) and by age at blood draw. Given the strong associations for many of the estrogens and estrogen metabolites evaluated with non-serous tumors, we evaluated the extent to which unconjugated estradiol (an estrogen strongly correlated with BMI among postmenopausal women) might explain the weak association between BMI and non-serous tumors. We compared the ORs for a $5 \mathrm{~kg} / \mathrm{m}^{2}$ increase in BMI, with and without further adjustment for unconjugated estradiol. We also conducted the following sensitivity analyses: 1) excluding individuals diagnosed within 2 years of blood draw $(n=14), 2)$ excluding potential outliers (greater than five standard deviations above the median; median excluded subject per hormone measure $n=7$ (min-max: $3-10)$ ), 3) excluding women who reported a history of diabetes at baseline ( $\mathrm{n}=29)$, and 4) excluding women who reported prior use of menopausal hormones $(\mathrm{n}=210)$. All $\mathrm{p}$-values were based on two-sided tests, for uncorrected tests $\mathrm{p}$-value $<0.05$ were considered statistically significant.

\section{RESULTS}

Characteristics of cases (overall and by subtype) and controls at baseline are presented in Table 1. Participants were on average 64 years of age at blood draw and predominantly white (90\%). Serous cases were slightly older, on average 64.7 years of age at blood draw compared to 63.2 years of age among non-serous cases. Median levels of estrogens and estrogen metabolites are compared by case-control status and subtype in Supplemental Table 1. Significantly higher levels of estrone and unconjugated 2-methoxyestrone were observed in ovarian cancer cases than controls. The median levels of the remaining estrogens and estrogen metabolites were slightly higher among ovarian cancer cases compared with controls, albeit differences were not statistically significant. There were no differences in median levels of the hormones comparing serous cases and controls. Significantly higher levels of estrone, unconjugated estradiol, and a number of other estrogen metabolites were observed in non-serous cases than controls.

Estrone was positively associated with overall ovarian cancer risk ( $\mathrm{p}$-trend $=0.05)$; however, the OR across extreme quintiles of estrone was not statistically significant (Q5 vs Q1: 1.54 (0.82-2.90)) (Table 2). Estradiol was not associated with overall ovarian cancer risk. Only 
two of the individual estrogen metabolites were significantly associated with overall ovarian cancer risk: compared with women in the lowest quintile, women with the highest quintile of 2-methoxyestrone were at increased risk of ovarian cancer (Q5 vs Q1: 2.03 (1.06-3.88); ptrend=0.02). A positive trend across quintile of 4-methoxyestrone was associated with risk (p-trend=0.01); however, the OR comparing extreme quintiles did not reach statistical significance (Q5 vs. Q1: $1.86(0.98-3.56))$. The ORs for these associations remained elevated in models adjusted for estrone; however, they no longer retained statistical significance (results not shown).

Significant heterogeneity in OR associations for the parent estrogens (estrone and estradiol) as well as the combined measurements of the 2-, 4-, and 16a-pathway metabolites was observed across histologic subtype of ovarian cancer (Figure 2). For serous ovarian cancers, associations with the estrogens and estrogen metabolites were universally null, while nonserous tumors were significantly associated with a number of individual estrogen metabolites (Table 3). In analyses restricted to non-serous cases, positive trends and/or significant top vs. bottom quintile OR associations were observed for 17 of the 25 individual estrogen measures evaluated. Estrone (2.65 (1.09-6.45), p-trend=0.01, pheterogeneity $=0.04)$ and unconjugated estradiol $(2.72(1.04-7.14), \mathrm{p}$-trend=0.03, pheterogeneity $=0.02$ ) were positively associated with non-serous tumors. For metabolites where the p-heterogeneity was $\leq 0.01$, non-serous ovarian cancer risk was at least tripled for the following: 2-hydroxyestrone (Q5 vs Q1:4.27 (1.52-12.01)), 2-methoxyestrone (Q5 vs Q1:4.26 (1.68-10.81), 16a-hydroxyestrone (Q5 vs Q1:3.33 (1.17-9.50)), and 16-epiestriol (Q5 vs Q1:3.46 (1.18-10.15)).

Some previous studies have shown associations of increased BMI with non-serous ovarian cancers. Accordingly, non-serous tumors were positively associated with BMI (OR (95\% CI) per $5 \mathrm{~kg} / \mathrm{m}^{2}$ increase in BMI: 1.21 (1.00-1.48), while serous tumors were not associated with BMI in the current study. Given the strong positive associations for many of the estrogens and non-serous tumors, and the known correlation between unconjugated estradiol and BMI, we evaluated the extent to which unconjugated estradiol might explain the association between BMI and non-serous tumors (Table 4). After adjustment for unconjugated estradiol, the association between BMI and non-serous tumors was substantially attenuated and no longer statistically significant (per $5 \mathrm{~kg} / \mathrm{m}^{2}$ increase in BMI: $1.10(0.88-1.38))$. The associations between unconjugated estradiol and non-serous tumors remained statistically significant after adjustment for potential confounding effects of BMI (OR (95\% CI) for a doubling in unconjugated estradiol without adjustment for BMI: 1.40 (1.13-1.73); and with adjustment for BMI: 1.36 (1.08-1.71)).

Results were not significantly modified by time between blood draw and diagnosis or age at blood draw (Supplemental Tables 2 and 3, respectively). In sensitivity analyses excluding cases diagnosed within two years of blood draw, excluding outliers for individual estrogen measurements, excluding individuals with a history of diabetes at baseline, or excluding former hormone users, effect estimates were largely unchanged (results not shown). 


\section{DISCUSSION}

There is strong in vitro and in vivo evidence, as well as some epidemiologic data, suggesting that estrogens (and potentially other sex steroids) play a role in ovarian carcinogenesis. However, previous studies did not show associations between circulating estrogens and overall ovarian cancer risk. In the current study, we demonstrate significant heterogeneity of associations between estrogen metabolites and ovarian cancer subtypes. We did not find associations with serous cancers, the most common and fatal subtype, and consequently, we did not see strong associations with overall ovarian cancer risk. However, for the first time, we provide evidence suggesting that estrogen and estrogen metabolites in postmenopausal women are associated with non-serous ovarian cancers.

In a previous study of early pregnancy hormones and subsequent cancer risk, Shock et al. (16) evaluated associations by histologic subtype and reported that higher levels of unconjugated estradiol in pregnancy were associated with increased risk of endometrioid ovarian tumors. They also reported elevated ORs (T3 vs T1 OR ranging from 1.54 to 1.80) for the association between estradiol and other non-serous subtypes including borderline and invasive mucinous and clear cell tumors, while ORs for serous tumors were null (OR borderline serous: 0.87; invasive serous: 0.98) (16). Although pregnancy levels of estrogens are high and may not correlate with non-pregnancy levels or levels among postmenopausal women, the increased risk associated with higher concentrations of the parent estrogens in our non-serous cases is consistent with the findings from the maternity cohort.

The three other prospective studies evaluating estrogens and overall ovarian cancer risk all reported null associations for the parent estrogens (13-15). This could be due to relatively limited number of available cases ( $\mathrm{n}=31$ (13), $\mathrm{n}=132$ across 3 cohorts (14), $\mathrm{n}=67$ (15)), but is most likely due to the predominance of serous tumors in these studies, as we observed null associations with parent estrogens and a very limited number of positive associations with estrogen metabolites in our analysis of overall ovarian cancer risk. In the only other study of estrogens metabolites to date, 4-methyoxyestrone was associated with a significant increased risk of overall ovarian cancer in a case-cohort study (p-trend $=0.04$ ), however the other 2-, 4- and 16-pathway metabolites evaluated were not significantly associated with overall risk (15). We note a similar elevated risk with 4-methoxyestrone in our overall ovarian cancer analysis ( $\mathrm{p}$-trend $=0.01$ ), which is likely driven, in part, by substantially elevated risk among non-serous tumors $(\mathrm{OR}=3.25)$. The findings from these early studies $(13,14)$ are not directly comparable to our results, given inclusion of pre- and perimenopausal women and measurement of parent estrogens using RIA, which have recognized limitations. While the findings from the recent case-cohort study are directly comparable, given measurement of estrogens using the same assay and that both study populations are primarily Caucasian, postmenopausal women with similar ages at blood draw and diagnosis (15).

The subtype-specific associations we observed in the current study are consistent with positive associations between BMI and non-serous tumors, and null associations between BMI and serous tumors, reported in prospective cohort studies (17-19). This is not surprising given that adipose tissue is a source of circulating estrogens via peripheral conversion of androgens in postmenopausal women (7). Ovarian cancer associations for 
other hormonal risk factors demonstrated more variability across the studies, but the extent to which these variables correlate with endogenous estrogen levels in postmenopausal women has not been well characterized. Associations with exogenous hormones, namely unopposed estrogen or estrogen plus progestin menopausal hormone therapy, suggest increased risks of both serous and non-serous subtypes $(17,18)$. Thus, the lack of association between circulating estrogens and serous tumors in the current study is at odds with results from the exogenous estrogen data. However, in women taking menopausal hormones, circulating levels of estrogens are severalfold higher than in postmenopausal women not currently taking exogenous hormones and have only limited applicability to the endogenous measures in the current study. Combined with the accumulating evidence of etiologic heterogeneity of epithelial ovarian cancers, particularly for hormonally-related factors, the subtype specific associations we observed in the current study support the evaluation of other circulating sex steroid hormones, namely androgens and progesterone, by ovarian cancer subtypes to further clarify the hormonal mechanisms that underlie the etiology of ovarian cancer.

The current study has several important strengths. The WHI-OS cohort is a large prospective study with standardized pre-diagnostic specimen collection and storage. The five estrogens and estrogen metabolites found in circulation in both unconjugated and combined forms and 10 measured in combined form provide a novel phenotypic characterization of individual patterns of estrogen metabolism. We were able to measure estrogens among all postmenopausal women in our study population, including those with relatively low levels, through the use of an LC-MS/MS assay with high sensitivity $(22,23)$. This study also has some limitations. Although we included all available cases, the study was still limited in power, which affected our ability to evaluate specific subtypes of non-serous tumors. While somewhat imprecise, the associations with non-serous tumors were robust to correction for multiple comparisons. We measured circulating estrogens in a single baseline serum sample, which may not accurately reflect long term exposure and/or intra-ovarian levels. Prior research by our group suggests that among postmenopausal women reproducibility was moderate-to-high for most of the estrogens and estrogen metabolites (e.g. 3 year ICCs greater than 0.65 and 0.72 for estrone and estradiol, respectively and on average greater than $0.25,0.29$, and 0.45 for the 2-, 4-, and 16-pathway metabolites, respectively) (24).

Our study provides novel molecular data that supports a role for estrogens and estrogen metabolites in non-serous ovarian cancer, and suggests etiologic heterogeneity across histologic subtypes of ovarian cancer. Further, the association of obesity with non-serous tumors appears to be explained, in part, by circulating estradiol. Additional investigation in a large prospective study is needed to clarify the risk of individual ovarian cancer subtypes for specific estrogen metabolites.

\section{Supplementary Material}

Refer to Web version on PubMed Central for supplementary material.

\section{Acknowledgments}

The authors would like to also acknowledge the following short list of WHI investigators: 
Program Office: (National Heart, Lung, and Blood Institute, Bethesda, Maryland) Jacques Rossouw, Shari Ludlam, Dale Burwen, Joan McGowan, Leslie Ford, and Nancy Geller

Clinical Coordinating Center: (Fred Hutchinson Cancer Research Center, Seattle, WA) Garnet Anderson, Ross Prentice, Andrea LaCroix, and Charles Kooperberg Investigators and Academic Centers: (Brigham and Women's Hospital, Harvard Medical School, Boston, MA) JoAnn E. Manson; (MedStar Health Research Institute/Howard University, Washington, DC) Barbara V. Howard; (Stanford Prevention Research Center, Stanford, CA) Marcia L. Stefanick; (The Ohio State University, Columbus, OH) Rebecca Jackson; (University of Arizona, Tucson/Phoenix, AZ) Cynthia A. Thomson; (University at Buffalo, Buffalo, NY) Jean Wactawski-Wende; (University of Florida, Gainesville/Jacksonville, FL) Marian Limacher; (University of Iowa, Iowa City/Davenport, IA) Robert Wallace; (University of Pittsburgh, Pittsburgh, PA) Lewis Kuller; (Wake Forest University School of Medicine, WinstonSalem, NC) Sally Shumaker

Women's Health Initiative Memory Study: (Wake Forest University School of Medicine, Winston-Salem, NC) Sally Shumaker

Financial support: This work was supported in part by the Intramural Research Program of the National Cancer Institute (B. Trabert, L.A. Brinton, R.M. Pfeiffer, R.T. Falk, N. Wentzensen). The WHI program is funded by the National Heart, Lung, and Blood Institute, National Institutes of Health, U.S. Department of Health and Human Services through contracts HHSN268201100046C, HHSN268201100001C, HHSN268201100002C, HHSN268201100003C, HHSN268201100004C, and HHSN271201100004C (G.L. Anderson, H.D. Strickler, L.H. Kuller, M.L. Gass).

\section{REFERENCES}

1. Lukanova A, Kaaks R. Endogenous hormones and ovarian cancer: epidemiology and current hypotheses. Cancer Epidemiol Biomarkers Prev. 2005; 14:98-107. [PubMed: 15668482]

2. Beral V, Gaitskell K, Hermon C, Moser K, Reeves G, Peto R. Menopausal hormone use and ovarian cancer risk: individual participant meta-analysis of 52 epidemiological studies. Lancet. 2015; 385:1835-1842. [PubMed: 25684585]

3. Trabert B, Wentzensen N, Yang HP, Sherman ME, Hollenbeck A, Danforth KN, et al. Ovarian cancer and menopausal hormone therapy in the NIH-AARP diet and health study. Br J Cancer. 2012; 107:1181-1187. [PubMed: 22929888]

4. Wentzensen, N.; Trabert, B.; Schildkraut, J. Ovarian Cancer. In: Goldman, MB.; Troisi, R.; Rexrode, KM., editors. Women and Health. Second. London: Elsevier Inc.; 2013. p. 1135-1145.

5. Yang HP, Anderson WF, Rosenberg PS, Trabert B, Gierach GL, Wentzensen N, et al. Ovarian cancer incidence trends in relation to changing patterns of menopausal hormone therapy use in the United States. J Clin Oncol. 2013; 31:2146-2151. [PubMed: 23650423]

6. Pike MC, Spicer DV. Hormonal contraception and chemoprevention of female cancers. Endocr Relat Cancer. 2000; 7:73-83. [PubMed: 10903525]

7. Erickson, GF.; Schreiber, JR. Morphology and physiology of the ovary. In: Becker, KL., editor. Principles and Practice of Endocrinology and Metabolism. Lippincott: Williams and Wilkins; 2001. p. 921-935.

8. Lippert TH, Seeger H, Mueck AO. The impact of endogenous estradiol metabolites on carcinogenesis. Steroids. 2000; 65:357-369. [PubMed: 10899535]

9. Nash JD, Ozols RF, Smyth JF, Hamilton TC. Estrogen and anti-estrogen effects on the growth of human epithelial ovarian cancer in vitro. Obstet Gynecol. 1989; 73:1009-1016. [PubMed: 2542854]

10. Syed V, Ulinski G, Mok SC, Yiu GK, Ho SM. Expression of gonadotropin receptor and growth responses to key reproductive hormones in normal and malignant human ovarian surface epithelial cells. Cancer Res. 2001; 61:6768-6776. [PubMed: 11559549]

11. Bai W, Oliveros-Saunders B, Wang Q, Acevedo-Duncan ME, Nicosia SV. Estrogen stimulation of ovarian surface epithelial cell proliferation. In Vitro Cell Dev Biol Anim. 2000; 36:657-666. [PubMed: 11229598]

12. Sieh W, Kobel M, Longacre TA, Bowtell DD, deFazio A, Goodman MT, et al. Hormone-receptor expression and ovarian cancer survival: an Ovarian Tumor Tissue Analysis consortium study. Lancet Oncol. 2013; 14:853-862. [PubMed: 23845225] 
13. Helzlsouer KJ, Alberg AJ, Gordon GB, Longcope C, Bush TL, Hoffman SC, et al. Serum gonadotropins and steroid hormones and the development of ovarian cancer. JAMA. 1995; 274:1926-1930. [PubMed: 8568986]

14. Lukanova A, Lundin E, Akhmedkhanov A, Micheli A, Rinaldi S, Zeleniuch-Jacquotte A, et al. Circulating levels of sex steroid hormones and risk of ovarian cancer. Int J Cancer. 2003; 104:636642. [PubMed: 12594820]

15. Dallal CM, Lacey JV Jr, Pfeiffer RM, Bauer DC, Falk RT, Buist DS, et al. Estrogen Metabolism and Risk of Postmenopausal Endometrial and Ovarian Cancer: the B FIT Cohort. Horm Cancer. 2016

16. Schock H, Surcel HM, Zeleniuch-Jacquotte A, Grankvist K, Lakso HA, Fortner RT, et al. Early pregnancy sex steroids and maternal risk of epithelial ovarian cancer. Endocr Relat Cancer. 2014; 21:831-844. [PubMed: 25270324]

17. Gates MA, Rosner BA, Hecht JL, Tworoger SS. Risk factors for epithelial ovarian cancer by histologic subtype. Am J Epidemiol. 2010; 171:45-53. [PubMed: 19910378]

18. Yang HP, Trabert B, Murphy MA, Sherman ME, Sampson JN, Brinton LA, et al. Ovarian cancer risk factors by histologic subtypes in the NIH-AARP Diet and Health Study. Int J Cancer. 2012; 131:938-948. [PubMed: 21960414]

19. Fortner RT, Ose J, Merritt MA, Schock H, Tjonneland A, Hansen L, et al. Reproductive and hormone-related risk factors for epithelial ovarian cancer by histologic pathways, invasiveness and histologic subtypes: Results from the EPIC cohort. Int J Cancer. 2015

20. Design of the Women's Health Initiative clinical trial and observational study. The Women's Health Initiative Study Group. Control Clin Trials. 1998; 19:61-109. [PubMed: 9492970]

21. Langer RD, White E, Lewis CE, Kotchen JM, Hendrix SL, Trevisan M. The Women's Health Initiative Observational Study: baseline characteristics of participants and reliability of baseline measures. Ann Epidemiol. 2003; 13:S107-S121. [PubMed: 14575943]

22. Xu X, Roman JM, Issaq HJ, Keefer LK, Veenstra TD, Ziegler RG. Quantitative measurement of endogenous estrogens and estrogen metabolites in human serum by liquid chromatography-tandem mass spectrometry. Anal Chem. 2007; 79:7813-7821. [PubMed: 17848096]

23. Loud JT, Gierach GL, Veenstra TD, Falk RT, Nichols K, Guttmann A, et al. Circulating estrogens and estrogens within the breast among postmenopausal BRCA1/2 mutation carriers. Breast Cancer Res Treat. 2014; 143:517-529. [PubMed: 24442642]

24. Falk RT, Brinton LA, Dorgan JF, Fuhrman BJ, Veenstra TD, Xu X, et al. Relationship of serum estrogens and estrogen metabolites to postmenopausal breast cancer risk: a nested case-control study. Breast Cancer Res. 2013; 15:R34. [PubMed: 23607871] 


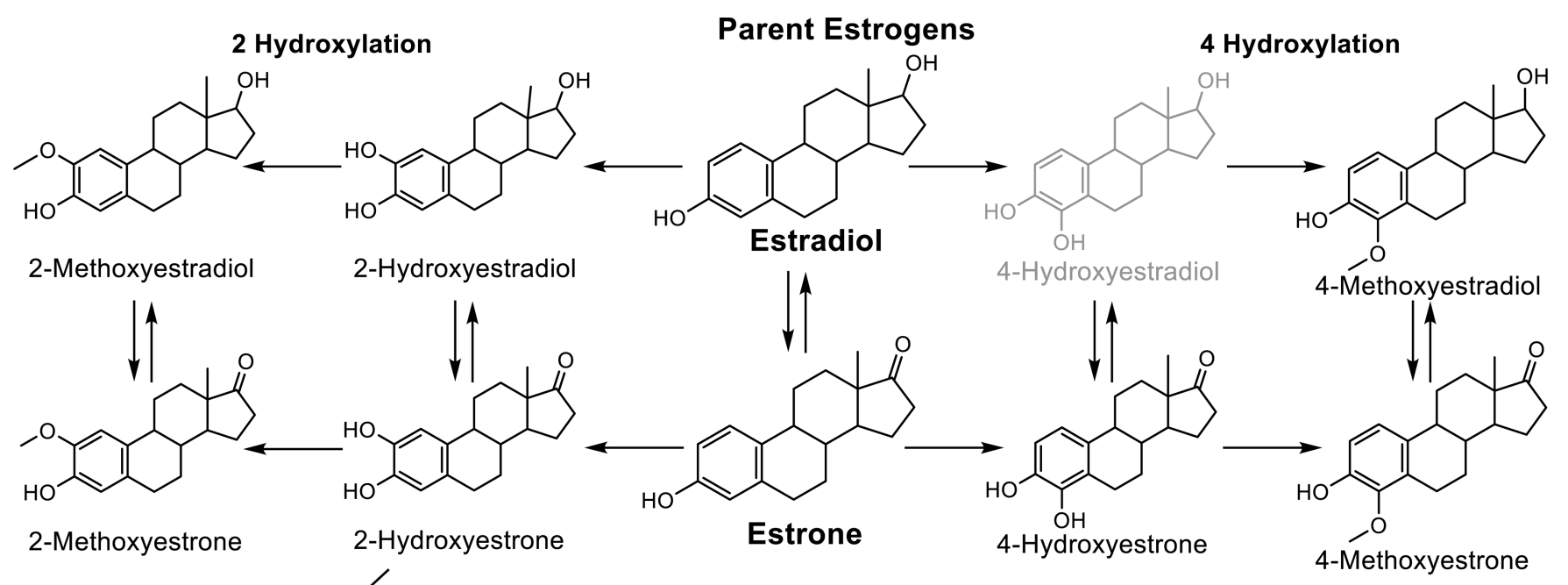<smiles>COc1cc2c(cc1O)C1CCC3(C)C(=O)CCC3C1CC2</smiles>

2-Hydroxyestrone-3methyl ether

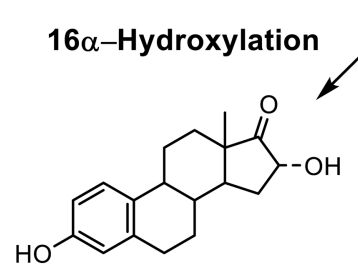

$16 \beta-H y d r o x y l a t i o n$

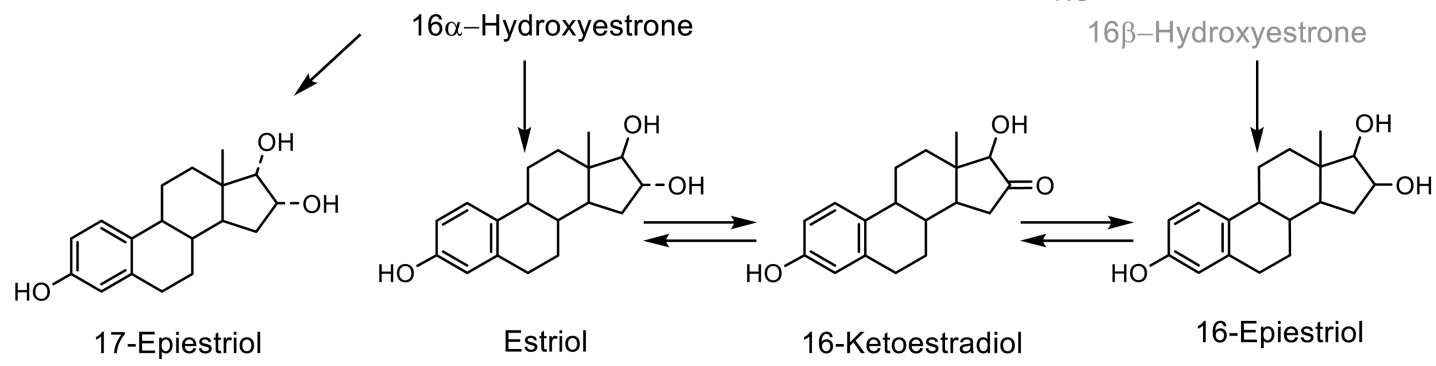

Figure 1.

Formation of 2-, 4-, and 16-hydroxylation pathway estrogen metabolites from parent estrogens. The current serum estrogen metabolite assay measures 15 of the 17 metabolites pictured; 4-Hydroxyestradiol and 16 $\beta$-Hydroxyestrone (in light gray) are not measured with the current assay due to very low abundance in circulation. 

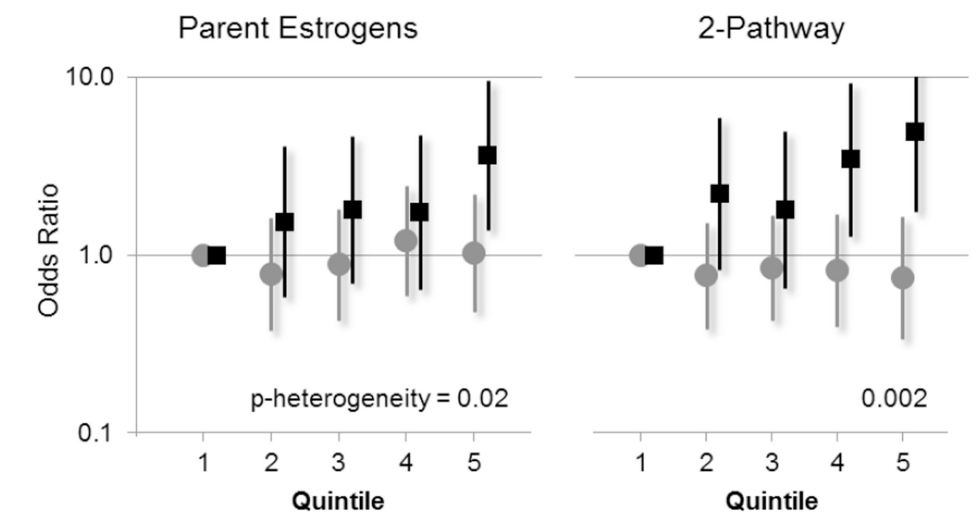

4-Pathway 16alpha-Pathway

Serous

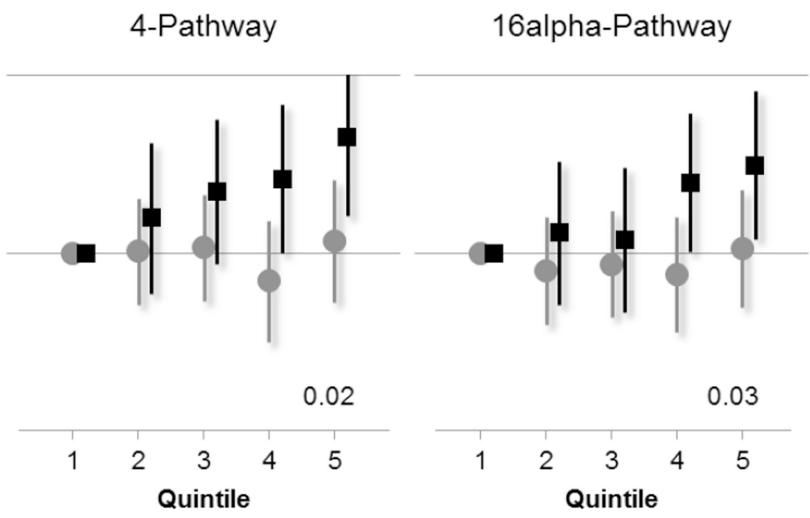

Figure 2.

Quintile specific odds ratios and 95\% confidence intervals for the risk of epithelial ovarian cancer subtypes with estrogen metabolite pathways, nested case-control study within the Women's Health Initiative Observational Study. 


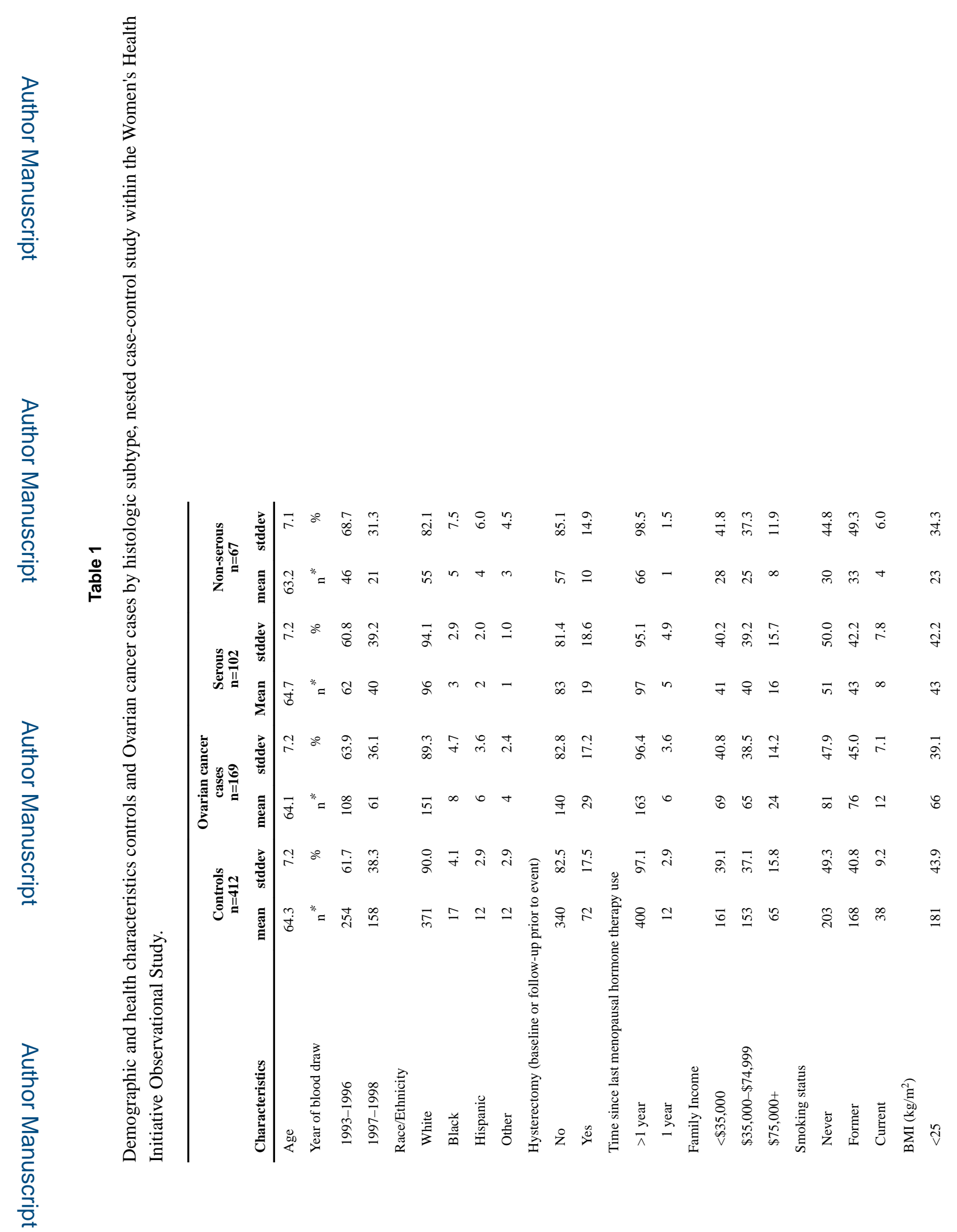

Cancer Epidemiol Biomarkers Prev. Author manuscript; available in PMC 2017 April 01. 


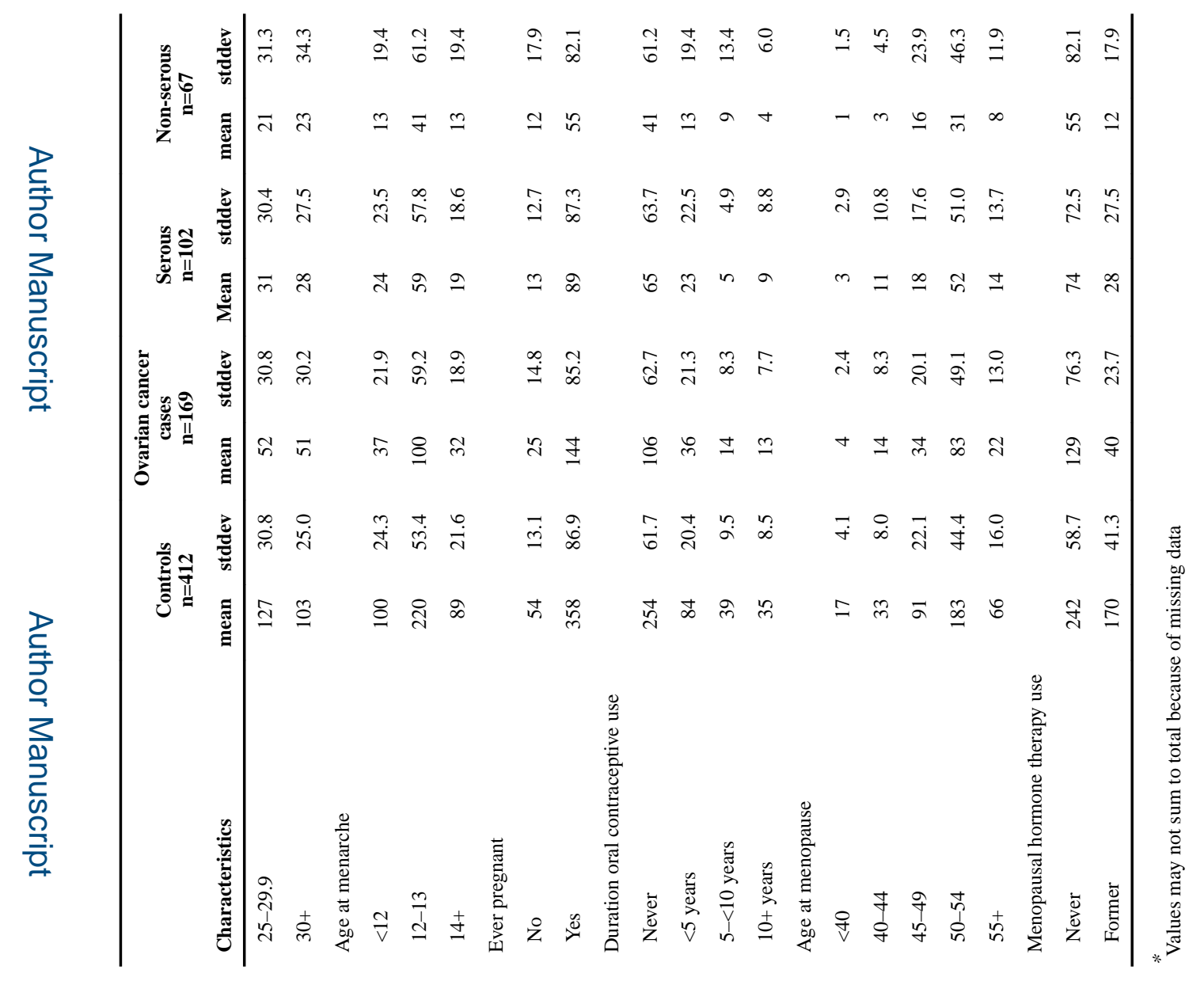

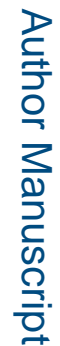

로을

Cancer Epidemiol Biomarkers Prev. Author manuscript; available in PMC 2017 April 01. 


\section{Table 2}

Odds ratios (OR) and 95\% confidence intervals (CI) for the risk of epithelial ovarian cancer comparing the 5th quintile to the 1st quintile for individual estrogens and estrogen metabolites, nested case-control study within the Women's Health Initiative Observational Study.

\begin{tabular}{|c|c|c|c|c|}
\hline Parent Estrogens & $\mathbf{O R}^{*}$ & $(95 \% \mathrm{CI})$ & p-trend ${ }^{\dagger}$ & FDR $^{f}$ \\
\hline Estrone & 1.54 & $(0.82-2.90)$ & 0.05 & 0.42 \\
\hline Unconjugated Estrone & 1.47 & $(0.78-2.79)$ & 0.25 & 0.48 \\
\hline Conjugated Estrone & 1.54 & $(0.81-2.92)$ & 0.09 & 0.42 \\
\hline Estradiol & 0.88 & $(0.47-1.62)$ & 0.89 & 0.95 \\
\hline Unconjugated Estradiol & 1.50 & $(0.77-2.91)$ & 0.39 & 0.54 \\
\hline Conjugated Estradiol & 1.10 & $(0.59-2.07)$ & 0.95 & 0.95 \\
\hline \multicolumn{5}{|l|}{ 2-Hydroxylation Pathway } \\
\hline 2-Hydroxyestrone & 1.31 & $(0.68-2.53)$ & 0.33 & 0.49 \\
\hline 2-Hydroxyestradiol & 0.93 & $(0.49-1.79)$ & 0.92 & 0.95 \\
\hline 2-Methoxyestrone & 2.03 & $(1.06-3.88)$ & 0.02 & 0.25 \\
\hline Unconjugated 2-Methoxyestrone & 1.44 & $(0.74-2.79)$ & 0.29 & 0.49 \\
\hline Conjugated 2-Methoxyestrone & 1.72 & $(0.90-3.26)$ & 0.10 & 0.42 \\
\hline 2-Methoxyestradiol & 1.55 & $(0.81-2.97)$ & 0.21 & 0.48 \\
\hline Unconjugated 2-Methoxyestradiol & 1.68 & $(0.88-3.18)$ & 0.21 & 0.48 \\
\hline Conjugated 2-Methoxyestradiol & 1.36 & $(0.72-2.58)$ & 0.30 & 0.49 \\
\hline 2-Hydroxyestrone-3-methyl ether & 1.11 & $(0.57-2.16)$ & 0.53 & 0.66 \\
\hline \multicolumn{5}{|l|}{ 4-Hydroxylation Pathway } \\
\hline 4-Hydroxyestrone & 1.46 & $(0.76-2.81)$ & 0.17 & 0.48 \\
\hline 4-Methoxyestrone & 1.86 & $(0.98-3.56)$ & 0.01 & 0.25 \\
\hline 4-Methoxyestradiol & 1.74 & $(0.90-3.40)$ & 0.13 & 0.46 \\
\hline \multicolumn{5}{|l|}{ 16a-Hydroxylation Pathway } \\
\hline 16a-Hydroxyestrone & 1.29 & $(0.68-2.45)$ & 0.32 & 0.49 \\
\hline Estriol & 1.61 & $(0.84-3.07)$ & 0.10 & 0.42 \\
\hline Unconjugated Estriol & 1.21 & $(0.62-2.36)$ & 0.46 & 0.61 \\
\hline Conjugated Estriol & 1.35 & $(0.70-2.58)$ & 0.19 & 0.48 \\
\hline 16-Ketoestradiol & 1.32 & $(0.70-2.50)$ & 0.24 & 0.48 \\
\hline 16-Epiestriol & 1.14 & $(0.58-2.24)$ & 0.92 & 0.95 \\
\hline 17-Epiestriol & 1.32 & $(0.66-2.63)$ & 0.68 & 0.81 \\
\hline
\end{tabular}

* OR from model conditioned on matching factors (age at baseline, year of blood draw, race/ethnicity, hysterectomy status, and time since last menopausal hormone use) and adjusted for body mass index, smoking status, gravidity, duration of oral contraceptive use, and never/former menopausal hormone therapy use.

${ }^{\dagger}$ p-values for trend across quintile (median value of category)

FDR q-value

Cancer Epidemiol Biomarkers Prev. Author manuscript; available in PMC 2017 April 01. 


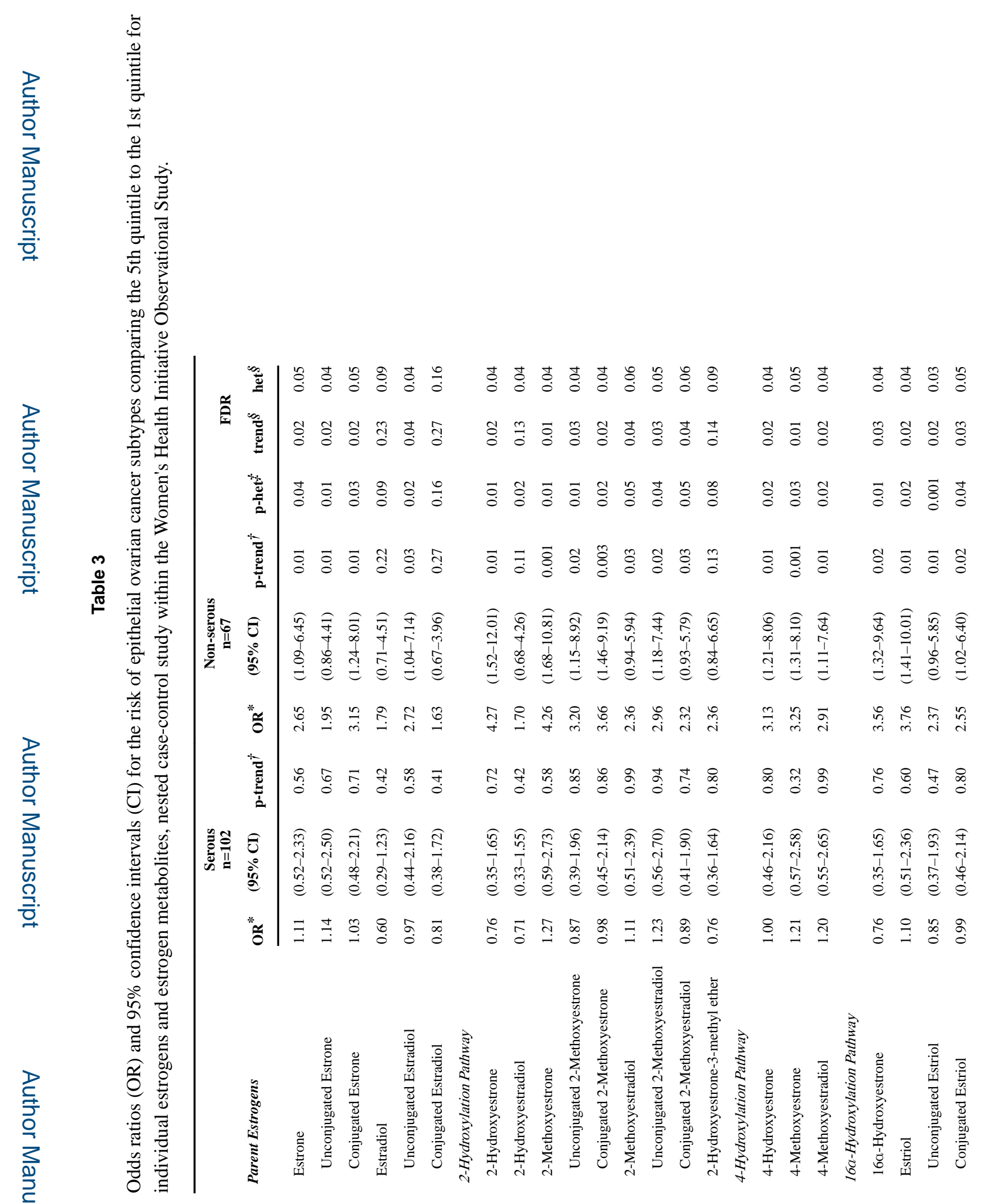

Cancer Epidemiol Biomarkers Prev. Author manuscript; available in PMC 2017 April 01. 


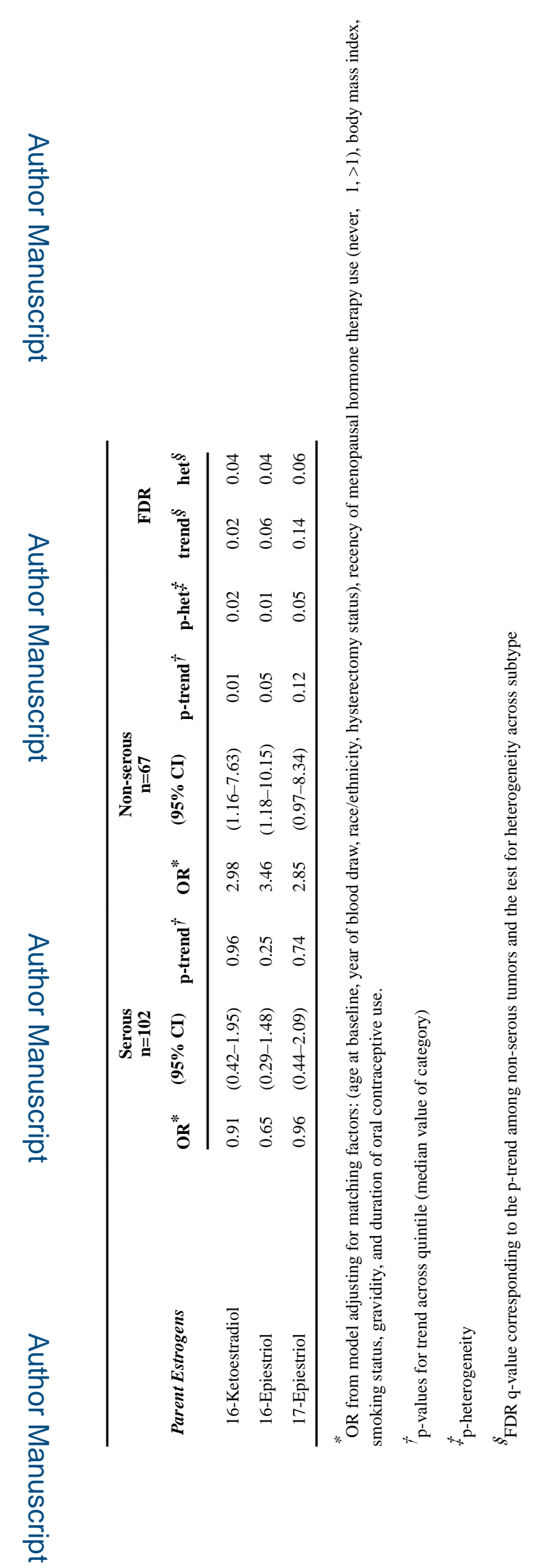

Cancer Epidemiol Biomarkers Prev. Author manuscript; available in PMC 2017 April 01. 


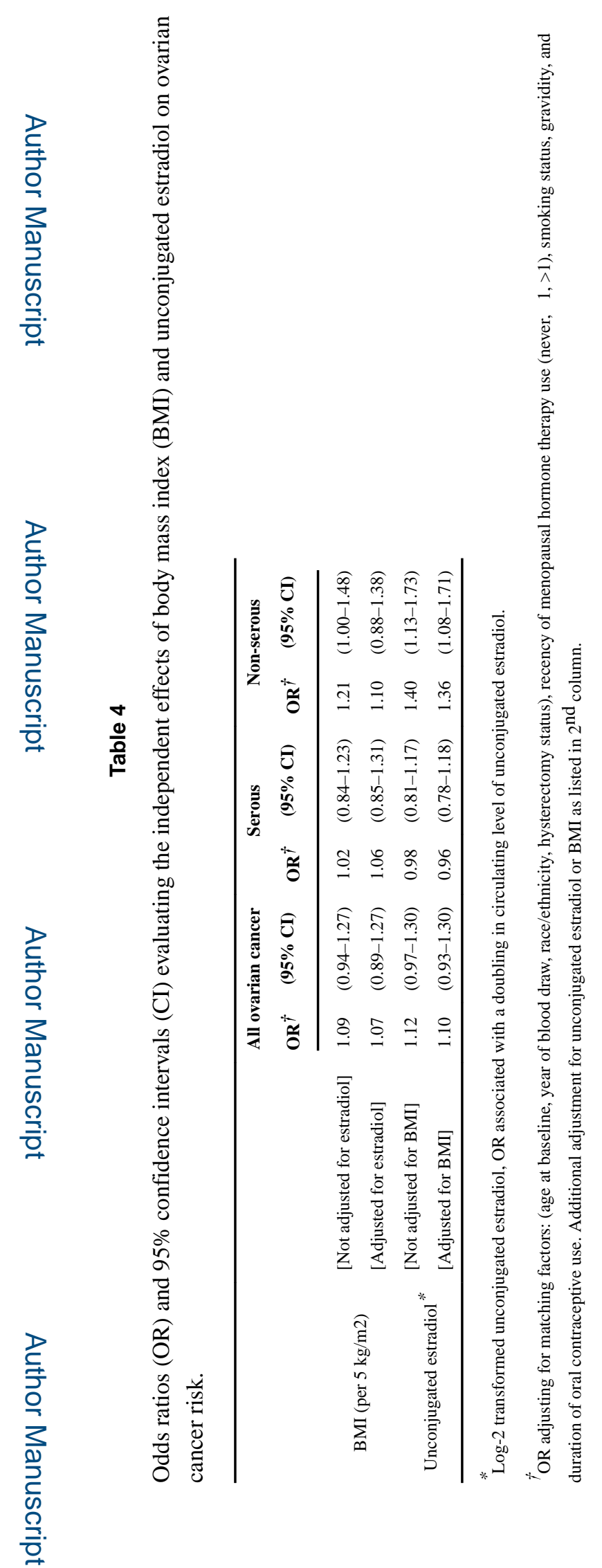

Cancer Epidemiol Biomarkers Prev. Author manuscript; available in PMC 2017 April 01. 